\title{
EL CULTIVO DEL RICINO Y EL AMBA'Y EN COMUNIDADES GUARANÍES DEL NORDESTE ARGENTINO, APROXIMACIÓN ETNOBOTÁNICA DE SU HISTORIA Y COSMOLOGÍA
}

\author{
HÉCTOR A. KELLER ${ }^{1}$, ANALÍA PIRONDO ${ }^{1}$ \& PABLO STAMPELLA²
}

\begin{abstract}
Summary: Keller, H. A., A. Pirondo \& P. Stampella. 2018. The cultivation of the castor bean and the amba'y in Guarani communities of the Argentine Northeast, ethnobotanical approach of their history and cosmology. Bonplandia 27(1): 23-30.

This work studied the historical evidence of the cultivation of "amba'y", Cecropia pachystachya (Urticaceae) in domestic areas of Guarani communities and its replacement by the castor bean or "ricino", Ricinus communis (Euphorbiaceae), whose cultivation is a current practice. Information obtained on uses of castor bean and on the mythical origin of the amba'y is useful to achieve an etymological approximation to the name "amba'y" alternative to those previously proposed by León Cadogan.
\end{abstract}

Key words: Cecropia, Ethnobotany, etymology, Ricinus.

Resumen: Keller, H. A., A. Pirondo \& P. Stampella. 2018. El cultivo del ricino y el amba'y en comunidades guaraníes del Nordeste Argentino, aproximación etnobotánica de su historia y cosmología. Bonplandia 27(1): 23-30.

Se estudian evidencias históricas sobre el cultivo del "amba'y", Cecropia pachystachya (Urticaceae) en ámbitos domésticos de comunidades guaraníes y su reemplazo por el "ricino", Ricinus communis (Euphorbiaceae), cuyo cultivo constituye aún una práctica corriente. Información obtenida sobre los usos del ricino y sobre el origen mítico del amba'y aportan información útil para lograr una aproximación etimológica al fitónimo "amba'y" alternativa a las propuestas previamente por León Cadogan.

Palabras clave: Cecropia, etimología, Etnobotánica, Ricinus.

\section{Introducción}

El término sucedáneo (del lat. succedaneus, sucesor, substituto) se aplica a la materia o sustancia que puede sustituir a otra por tener propiedades semejantes a ella, según la Real Academia Española. En botánica económica es frecuente que tal sustitución se efectúe debido a la escasez de una determinada especie útil, como es el caso de los sucedáneos de Quassia amara L. (Simaroubaceae), como también por su similitud morfológica y asignación de igual nombre vernáculo, como ocurre en el caso de las "canchalaguas" (Correa, 2003; Di Sapio et al., 2012).

${ }^{1}$ Instituto de Botánica del Nordeste - CONICET, Casilla de Correo 209, 3400-Corrientes, Argentina. E-mail: ibone@ agr.unne.edu.ar

${ }^{2}$ Laboratorio de Etnobotánica y Botánica Aplicada (LEBA). Facultad de Ciencias Naturales y Museo, Universidad Nacional de La Plata, Calle 64 nro. 3, 1900-La Plata, Argentina. Becario CONICET. E-mail: pstampella@yahoo.com 
La adopción de plantas sucedáneas es una práctica frecuente dentro de comunidades inmigrantes, quienes traen en su memoria colectiva conocimientos morfológicos y benéficos de etnoespecies naturales de sus comarcas de procedencia; pero que al no traer consigo diásporas de todas ellas, suelen recrear con la mayor fidelidad posible su escenario etnobotánico original a partir de la adopción de especies útiles sustitutas, con frecuencia similares a aquellas en su morfología o en sus beneficios. Abundan los ejemplos de esto durante la época jesuítica: en cuanto a la morfología Dobrizhoffer (1967) compara el "Quabyra guazu" [Campomanesia xanthocarpa (Mart.) O. Berg, Myrtaceae] y el "Quabiyú" [Myrcianthes pungens (O. Berg) D. Legrand, Myrtaceae] con ciruelas y cerezas, respectivamente; a la "Granadilla" o "Pasionaria" (Passionaria spp., Passifloraceae) con la "granada" (Punica granatum L., Punicaceae); al "Tatayy" con la "morera" (Morus sp., Moraceae); y más raramente a las "bananas" con las "higueras": "Las frutas bacoba y banana, tan altamente apreciadas por los americanos, pertenecen al género de los higos" (Dobrizhoffer, 1967: 487); mientras que Montenegro (2009) se refiere a M. pungens y a Eugenia uniflora L. (Myrtaceae) como "Arrayanes negro y blanco", respectivamente, o a los "aguarandios" (Piper spp., Piperaceae) con el "Asaro" de Dioscorides (Asarum europaeum L., Aristolochiaceae), el "caáné" [Dysphania ambrosioides (L.) Mosyakin \& Clemants, Amaranthaceae) con el "Lepidio" (Lepidium latifolium L., Brassicaceae) de Plinio.

En el contexto de la fitomedicina tupiguaraní un pueblo que desde la selva amazónica se ha expandido hacia otras regiones selváticas tropicales y subtropicales de Sudamérica, se ha sugerido que la adopción de sucedáneos vegetales a lo largo de sus procesos expansivos, ha contribuido ampliamente al desarrollo de su farmacopea (Keller, 2007a), siendo también una práctica frecuente en relación a otros tipos de usos, como las plantas mágicas (Keller, 2011) y los ictiotóxicos (Keller, 2008a). A resultas de esto, en la actualidad fitónimos guaraníes equivalentes y usos idénticos para especies similares, son frecuentes en regiones distantes dentro del área de distribución TupiGuaraní (Balée \& Cebolla Badie, 2009).

En este trabajo se aborda la adopción de Ricinus communis L. (Euphorbiaceae) como sucedáneo de Cecropia pachystachya Trécul (Urticaceae) entre comunidades de filiación guaraní de Misiones y Corrientes (Argentina). El estudio de caso sugiere que conocimientos ancestrales sobre un vegetal que se han modificado en relación a dicha especie, aún pueden recuperarse estudiando la etnobotánica actual e histórica de especies sustitutas. Asimismo el tratamiento de este caso aporta elementos para dilucidar una posible interpretación etimológica del fitónimo "amba'y".

\section{Materiales}

Cecropia pachystachya. ARGENTINA. Misiones: Dep. Guaraní, Predio Guaraní, $26^{\circ}$ $54^{\prime}-59^{\prime} \mathrm{S}$ y $54^{\circ} 12^{\prime}-18^{\prime} \mathrm{W}$, camino a arroyo Soberbio, 12-II-2002, Keller \& Robledo 1618 (CTES).

Ricinus communis. ARGENTINA. Misiones. Dep. Guaraní, Predio Guaraní, $26^{\circ}$ 54'-59'S y 54 ${ }^{\circ} 12^{\prime}$ '-18'W, aldea aborigen, 07II-2001, Keller 607 (CTES).

\section{Resultados}

\section{Ricinus communis}

El ricino o tártago, $R$. communis, es un arbusto o arbolito con tallos gruesos, fistulosos, externamente adornados de cicatrices foliares anulares. Sus hojas son glabras, largamente pecioladas, con láminas palmatipartidas de lóbulos aserrados. Es originario de las zonas tropicales del este de África y tempranamente se ha cultivado y naturalizado en regiones cálidas de todo el mundo. Su presencia en América fue ya registrada en el siglo XIV por Fernández de Oviedo (1535) para La Española (República Dominicana-Haití), isla que constituyó el primer asentamiento europeo en "el nuevo mundo".

Los jesuitas de la cuenca AsunceñaRioplatense mencionan de manera escueta a esta planta denominándola "tártago", "resino 
árbol”, “ambaí guazú”, “ambaybuzu”, “pinó” o "higuera del infierno", siendo una de las "nueces purgantes" o "nuces catharticae". Montenegro (2009: 293-294) es quien se detiene a describir esta planta indicando algunas de sus propiedades: “(...) es el recino blanco de Plinio, que crece su arbol como el de la higuera, y las ojas son algo parecidas en el grandor; pero no en su aspereza y sequedad, y azabaladuras, porque son lisas y muy hermosas á la vista, adornadas de un verde claro, y muy tersas, con bastante humedad: nace por las orillas de los bosques, $y$ huertos, á donde la tierra es fértil y humeda: y cierto, que si bien se mira es la mejor de las especies de recino, ó tártago, á que el vulgo llama Higuera del Infierno, y aunque de esta especie hay variedad, asi en la figura como en el grandor de sus granos y oja: el uso de medicina me atengo mas á este, que no á ninguno de todas las otras diferencias, por no ser tan venenoso, enemigo del estomago, no causando tantas congojas, ni nauceas, como el tartago mayor ordinario. (...) Se saca aceite de sus semillas, el cual sirve asi para lamparas, como para el uso de medicina, y asi tomadas diez ó dice gotas de él en caldo de pollo, purga suavisimamente la flema, y la colera. Veinte de sus granos mondados metidos dentro de un gallo viejo, y bebido su caldo, es único remedio en el mal de hijada, y en el dolor de la esciatica, y en el dolor de las coyunturas por humores frios y galicos. Sus ojas, esto es, de todos los racimos, machacadas y cocidas, y aplicadas á modo de emplasto extirpan los barros, y las manchas del rostro, y de todo el cuerpo, asi las que llaman albarazos, como de los asoleados: aplicadas su ojas con polenta reprimen las inchazones, y inflamacion de los ojos, y aplicandolas calientes, ó mojadas en vinagre á los pechos endurecidos, las relaja, y resuelve la demaciada leche: asi mismo, mojadas en vinagre caliente ataja el fuego de S.n Anton, y de S.n Torcáz". Esta descripción fue tomada por el autor casi textualmente de la obra de Andrés Laguna (ver Font Quer, 1993).

Paucke (1944: 188) también menciona el uso del aceite de ricino para alumbrado, especialmente por los portugueses de Brasil, y las propiedades medicinales de sus hojas para curar la erisipela. Por otra parte Dobrizhoffer
(1967: 463) describe una planta similar, posiblemente Cascabela thevethia (L.) Lippold (Apocynaceae) o alguna especie del género Jatropha (Euphorbiaceae) debido a la presencia de semillas carunculadas, denominándola ricini americani debido a su semejanza en su uso purgante.

Azara (1998) se refiere al "tártago", aclarando que en América se lo nombra "palma-christi", como una planta que nadie cultiva, pero que..."se la encuentra siempre al lado de las casas, de las granjas o de los jardines, y yo no me acuerdo de haberla visto en los desiertos, lo que hace pensar que es del número de las que crecen donde hay hombres. Se encuentra en todos los parajes y poblados". De igual manera D'Orbigny (1945) en su obra "Viaje a la América Meridional" destaca la presencia del "ricino" o "palma-christi" y lo ubica en los sitios donde los indígenas fijaban su residencia momentánea durante las campañas militares. Aludiendo además que “(...) es un indicio que no engaña nunca, es el compañero fiel del hombre en sus migraciones, lo sigue a todas partes y que no brota lejos de él. Es necesario que éste prepare la tierra donde esa planta debe vivir".

En la actualidad, la especie se encuentra naturalizada en la Argentina en diversas provincias del centro y norte del país (Bacigalupo \& Múlgura, 1999). Sus poblaciones rápidamente han proliferado por regeneración natural marcando una impronta notable en la flora ruderal de varias localidades. Constituye un ejemplo de ello la conformación de topónimos "Tartagal", correspondientes a un municipio de la provincia de Salta y también a una localidad del Dep. Santo Tomé, Corrientes.

Los fitónimos más corrientes para nombrarla en contextos urbanos y rurales del país son "tártago" (nombre dado al ricino por el vulgo castellano) y "ricino" (del lat. ricinus: garrapata, aludiendo a la similitud de las semillas con estos ectoparásitos) (Font Quer, 1993).

En comunidades guaraníes contemporáneas de Misiones y Corrientes la especie es denominada $a m b a$ ' $y$, y es muy frecuentemente cultivada en el entorno de las viviendas (Fig. $1 \mathrm{~A}$ ver flecha) como árbol de sombra y para 

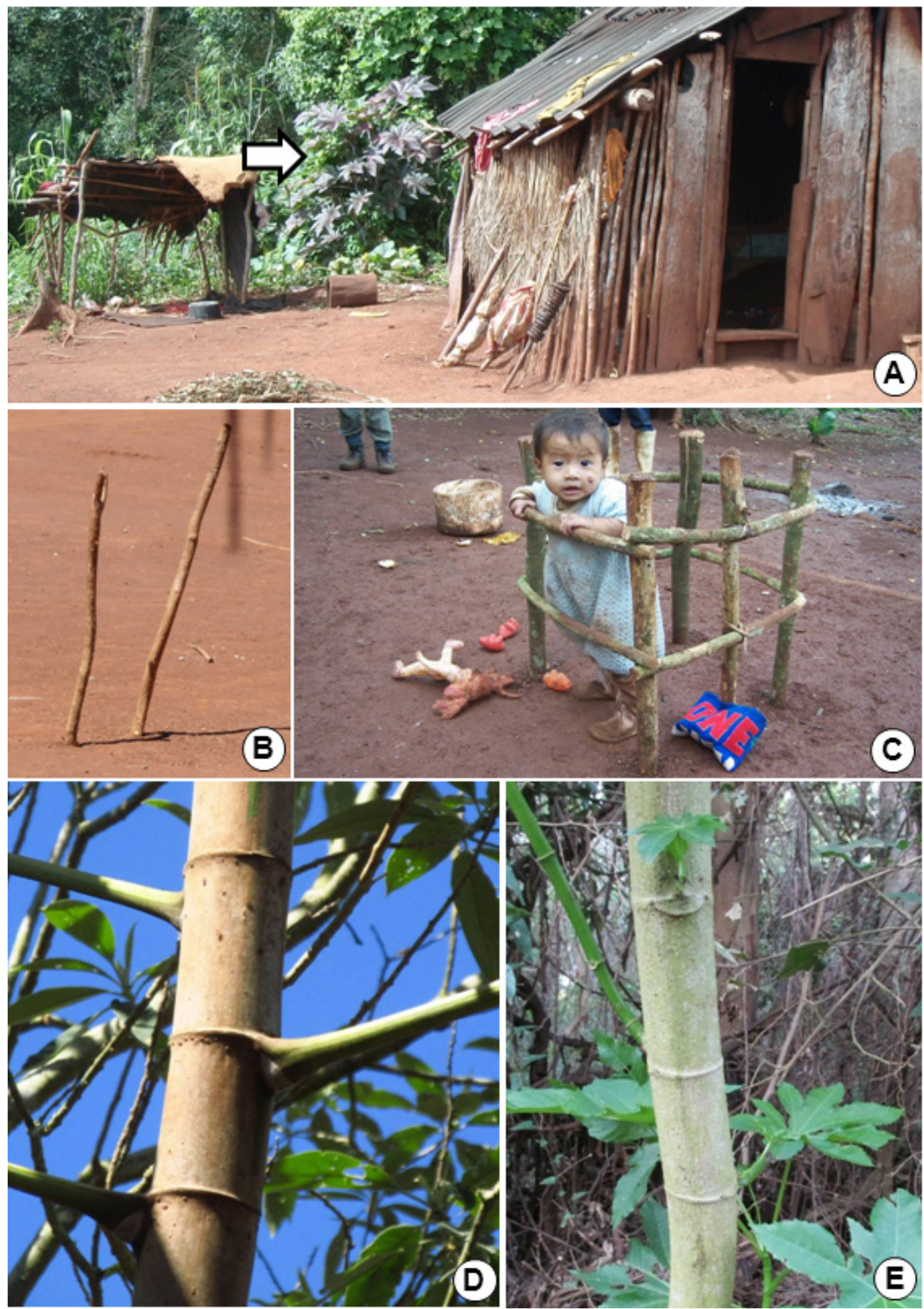

Fig. 1. A: Ricino cultivado junto a vivienda guaraní. B: Amba de dos varas frente a vivienda guaraní. C: Amba tipo corralito. D: Tallo con nudos notables de Cecropia pachystachya. E: Tallo con nudos notables de Ricinus communis. Fig. 1. A: Castor bean growing near to the guarani house. B: Amba of two stems at the head of guarani house. C: Amba like a small yard. D: Stem with conspicuous nodes of Cecropia pachystachya. E: Stem with conspicuous nodes of Ricinus communis. 
aprovechar sus virtudes medicinales, llegando a ser un árbol infaltable en el patio de las aldeas (Keller, 2008b; Pirondo, 2016).

Interlocutores guaraníes Mbya de la comunidad Pindoty, San Ignacio, Misiones sugieren que por el sólo hecho de cultivarse esta especie en el contexto hogareño los niños aprenden más tempranamente a erguirse y caminar, constituyendo esta la razón fundamental por la que es un elemento infaltable en los patios de las viviendas.

\section{Cecropia pachystachya}

Es un árbol similar al ricino por sus tallos fistulosos, externamente ornamentados con cicatrices foliares anulares; hojas largamente pecioladas con láminas palmatipartidas. No obstante, entre otras diferencias, alcanza mayor altura que el ricino (hasta $15 \mathrm{~m}$ ) y sus láminas foliares son escabrosas en el epifilo y cubiertas de pelos lanosos blancos en el hipofilo; por su parte los lóbulos del limbo foliar no presentan hendiduras superficiales.

La especie ha sido registrada en Brasil, Paraguay y la Argentina (Zuloaga et al., 2008). Es más frecuente la conformación de poblaciones densas en bosques riparios y constituye una especie pionera en sucesiones secundarias luego de la tala o la apertura del dosel selvático por caída de árboles.

Los jesuitas tardíos (siglo XVIII) mencionan el cultivo de esta planta en los alrededores de las chozas de los indios, siendo estimada como ornamental, medicinal y para iniciar fuego: “(...) del género de las higueras silvestres (...) que los Indios plantan de buen grado estos árboles en derredor de sus chozas porque saben aprovecharlos de diversas maneras. Secan sus raices y luego restriegan contra ellas unos palitos de madera dura lo más rápidamente posible para producir (...) el fuego que luego captan con cáñamo, papa o una hoja seca (...). Además el ambay no sólo deleita la vista por su ameno color verde sino que es muy estimado (...) por su fuerza medicinal que ofrecen su corteza, hojas y juego (jugo) en la gonorrea, menorragia y disentería (Dobrizhoffer, 1967: 503). Sánchez Labrador la diferencia del ricino ("ambaybuzu") denominándola "ambay caaimbe guazú" (Furlong, 1948).
En la literatura farmacobotánica del país la especie es medicinal, constituyendo sus propiedades antiespasmódicas del sistema respiratorio, hipotensoras y antimicrobianas sus virtudes más reputadas (Alonso, 2004). En la vernácula de su área de distribución y en la farmacopea popular argentina el fitónimo más utilizado para denominar a esta especie es ambay (Saggese, 1959; Ratera \& Ratera, 1980; Toursarkissian, 1980). En el contexto de los grupos guaraníes este nombre corresponde a C. pachystachya en los registros de fitonimia (Bertoni, 1940; Cadogan, 1957, 1973, 1992), sin embargo en las aldeas Mbya y Ava Chiripa de Misiones actualmente recibe el nombre "amba'y guachu” (amba'y de selva), expresión que permite diferenciarla del ricino, llamado amba'y okare (amba'y del patio) o simplemente amba'y (Keller, 2008c).

\section{Un árbol posdiluviano}

Yy typa ve rire eta omano va'ekue itui yvy pyau ary. Ñanderu ombou yryvu ypy omboguejy angua tatachina ombovy ju angua. A'e va'ekuery oguapy amba'y ypy raka ary. A'upei ma vy vy ouma ñanderu oma'e vy aevyma oecha tatachi petyngua mapo ei aepy oechama yryvu oechyramo teongue aepy ombojerama yryvu chi aevyma ombou yryvu iñaka pyta vae ju

"Cuando las aguas bajaron innumerables cadáveres estaban tendidos en la nueva tierra. A quienes más tarde serían transformados en aves carroñeras se les encomendó resucitar a los muertos dotándoles a sus pipas con tatachina (niebla creativa). Para hacerlo se apostaron sobre las ramas del árbol del amba primigenio. Cuando el creador se acercó a los jotes blancos observó que el humo no era el de sus pipas, más bien estaban asando a los cadáveres para alimentarse, entonces los transformó en aves carroñeras y la tarea fue encomendada a los futuros jotes de cabeza roja.... (fragmento de una versión del mito diluviano guaraní)".

Este fragmento posdiluviano de los Ava Chiripa de Misiones sugiere que el amba'y (árbol del amba) sobre el cual se apostaron los encargados de la resurrección, es el primer vegetal de la nueva tierra que se conformó luego de uno de los tantos cataclismos presentes 
entre los mitos escatológicos guaraníes, la gran inundación. Cecropia pachystachya es una especie con demostradas aptitudes fisiológicas y morfoanatómicas para tolerar períodos de inundación, por ejemplo los individuos anegados manifiestan un aumento y acondicionamiento de lenticelas para favorecer el intercambio gaseoso en condiciones de hipoxia, mayor producción de raíces adventicias provistas de tejido aerenquimático y una satisfactoria producción energética posiblemente a través de la aceleración de la glicólisis, entre otras adaptaciones (Batista et al., 2008). Tales atributos hacen plausible ese rol mítico primigenio en la sucesión vegetal, por lo que, si bien los guaraníes actuales utilizan la expresión amba'y (árbol del amba) para referirse al tártago o ricino, es más probable que la narrativa refiera en sus inicios a C. pachystachya.

\section{¿Qué es el ãmba?}

Entre los guaraníes se conoce como amba a un dispositivo doméstico que propicia el ejercicio infantil de ponerse de pie y dar los primeros pasos. Se trata de dos o más varas de madera de unos pocos centímetros de diámetro y cerca de un metro de altura, cuyos extremos basales se entierran firmemente en el suelo (Fig.1B). Los niños pequeños suelen llegar gateando o arrastrándose hasta el $a m b a$, y se los adiestra para que se aferren a estas varas e intenten erguirse y luego, dar los primeros pasos agarrándose a otras varas situadas lateralmente. Algunos amba actuales se conforman como cercos de contención infantil (Fig. 1C) pero es muy probable que se esté tratando de emular el "corralito" o cuna que utilizan los juru'a (los muchos) para ocuparse de sus quehaceres mientras los niños permanecen encerrados.

La expresión amba puede desagregarse en dos vocablos componentes que le dan sentido a su empleo para hacer referencia a dicho dispositivo: ã-mba (erguirse completamente). La partícula $m b a$ (completamente) es un sufijo de compleción que se utiliza para denotar que un conjunto de cosas o un evento alcanza su plenitud o totalidad. El primer vocablo ã, sobre el cual oportunamente se detiene Cadogan (1992) en su diccionario, quiere decir “estar en posición vertical", "erguirse", "vivir".
Añadiendo algunos párrafos en lengua Mbya que ejemplifican las consecuencias de no ser beneficiario del atributo de verticalidad. " $P o$ ' $\tilde{a}$ " (medicina) se desagrega en po-ã (propiciadores de la condición erguida), es decir las sustancias medicinales son concebidas como aquello que nos restituye la posición erguida. "Che ra'y o' ã'ey va'ekue", "mi hijo que murió prematuramente" (mi hijo que no se irguió adulto) (p. 15).

$\mathrm{Si}$ hacemos una relectura del relato posdiluviano bajo la lente de estas connotaciones complementarias de la condición erguida (ã) que atesora la lengua guaraní, se puede entender mejor el rol del "árbol del $a m b a$ " en la resurrección del mundo y de la humanidad después de un evento escatológico. Y si con dicha relectura retornamos nuevamente al ámbito doméstico se percibe con mayor claridad la trascendencia del "amba" erigido en los patios, un dispositivo que posibilita al niño superar la condición de horizontalidad propia de los fenecidos y proyectarse hacia la vida.

Amba'y: el árbol de las varas para erguirse

El sufijo " $y$ " se emplea en fitonimia guaraní para designar fustes leñosos, ya sean palos, varas, madera o los árboles en general (Cadogan, 1992; Keller, 2013), por lo que es muy probable que las ramas de Cecropia pachystachya se usen o hayan usado como material para erigir los amba en los patios de las viviendas. La formación de nudos notables en los vástagos leñosos (Fig. 1D), debido a las grandes cicatrices foliares, propicia un asidero propicio para los niños que intentan erguirse aferrándose a estas varas. Este atributo es compartido con el ricino, cuyos tallos también presentan nudos notables (Fig. 1E) y probablemente también hayan sido usados para este fin.

La presencia de ejemplares de $C$. pachystachya como elemento pionero de las sucesiones secundarias luego de la roza y quema en el entorno de las comunidades ha sido un argumento utilizado por Cadogan (1957) para sugerir una posible raíz etimológica de la expresión amba'y proponiendo "árbol de la morada" como un posible significado etimológico (p. 23). En otra contribución (Cadogan, 1973) sugiere el atributo de similitud entre las hojas palmatipartidas de ambas 
especies para explicar las razones por las cuales los misioneros echaron mano de la expresión amba'y ete (verdadero amba'y) para nombrar al ricino (p. 14).

Es muy común que en este tipo de especies pioneras se obtengan de forma agámica nuevas plantas a partir de estacas plantadas en el suelo (ej. para erigir un $a m b a$ ), lo que quizás explique la práctica de cultivar $C$. pachystachya en los patios que fue documentada para los guaraníes en el siglo XVIII. Actualmente no se observa el cultivo de dicha especie en las comunidades, en cambio el amba'y que con frecuencia se cultiva en los patios como procedimiento para que los niños caminen a más temprana edad es $R$. communis, sucedáneo con atributos morfológicos y utilitarios equivalentes.

\section{Discusión y Conclusiones}

Los documentos históricos permitieron recopilar fitónimos de estas plantas como también agrupamientos de las mismas. Se observó que los guaraníes consideran tanto a $C$. pachystachya y a $R$. communis dentro del grupo de los amba'y, mientras que los jesuitas, debido a su legado occidental, consideraban a las mismas dentro de la categoría de los ricinos.

Las aproximaciones etimológicas a la expresión amba'y propuestas por Cadogan (1957, 1973, 1992), "árbol que se yergue", "árbol de la morada", "árbol de los lugares habitados por seres erectos", han resultado difusas o muy complejas por carecer de la acepción "amba" como dispositivo para que los niños aprendan a erguirse y caminar. Sugerimos aquí el significado "varas para erigir el $a m b a "$ como una opción etimológica alternativa a las propuestas por dicho autor.

A lo largo del contacto entre proyectos evangelizadores y pueblos guaraníticos ha sido de usufructo corriente el sincretismo dirigido, es decir la inscripción de nociones del cristianismo en elementos de la naturaleza que son centrales a la cosmología del pueblo que se ha deseado convertir. De esta manera, Tupã el progenitor de los rayos y deidad guaraní de la guerra constituyó una entidad idónea para inculcar la noción del dios cristiano (Keller, 2017). En la cosmología Mbya guaraní el ejemplar primigenio de la yerba mate, Ilex paraguariensis A. St.-Hil. (Aquifoliaceae) surgió de la transformación de una mujer castigada por no acceder a propuestas matrimoniales (Keller, 2007b); en la versión de Ambrosetti (1893) recogida entre los guaraníes cristianizados se conoce con el nombre de María a dicha mujer y su transformación en una planta constituyó un premio por mantenerse hasta cierta edad avanzada alejada de los pecados carnales.

En estos dos ejemplos el "sincretismo dirigido" involucra el reemplazo o la tergiversación, con conocimiento de causa, de nociones asociados a elementos de la naturaleza que cuentan con cierta relevancia cosmológica. En el caso que aquí se presenta se da una situación inversa, ya que se verifica el reemplazo de una especie por otra sucedánea, la que pasa a ser la depositaria de los constructos cosmológicos asociados a la primera. Ello nos permite sugerir que los conocimientos que un grupo étnico mantiene y recrea en torno a un sustituto vegetal pueden aportar elementos para entender mejor su relación ancestral con la especie substituida, así como también, en este caso particular, echar luz sobre la etimología del fitónimo con que se la designa.

\section{Bibliografía}

ALONSO, J. 2004. Tratado de Fitofármacos y Nutracéuticos. Rosario: Ed. Corpus. 1359 pp.

AMBROSETTI, J. B. 1893. Materiales para el estudio del Folklore Misionero. Revista del Jardín Zoológico de Buenos Aires 1: 129-160.

AZARA, F. 1998 [1809]. Viaje por la América Meridional, Tomo I. Ed. Elefante Blanco: Buenos Aires. 455 pp.

BACIGALUPO, N. M. \& M. E. MÚlgURA. 1999. Euphorbiaceae. En ZULOAGA, O. \& O. MORRONE (eds.) Catálogo de las Plantas Vasculares de la República Argentina II. Acanthaceae-Euphorbiaceae (Dicotyledoneae). Missouri Botanical Garden Press, St. Louis, pp. 590-622.

BALÉE, W. \& M. CEBOLLA BADIE. 2009. The Meaning of "Tree" in Two Different Tupí-Guaraní Languages from Two Different Neotropical Forests. Amazônica 1: 96-135.

BATISTA, C. U. N., M. E. MEDRI, E. BIANCHINI, C. MEDRI \& J. A. PIMENTA. 2008. Tolerância à inundação de Cecropia pachystachya (Cecropiaceae): aspectos ecofisiológicos e morfoanatômicos. Acta Bot. Bras. 22: 91-98. 
BERTONI, M. S. 1940. Diccionario Botánico LatinoGuaraní; Guaraní-Latino. Asunción: Ed. Guaraní. $156 \mathrm{pp}$.

CADOGAN, L. 1957. Breve contribución al estudio de la nomenclatura guaraní en Botánica. Asunción: Ministerio de Agricultura y Ganadería. Servicio Técnico Interamericano de Cooperación Agrícola. Boletín 196:1-49.

CADOGAN, L. 1973. Ta-ngy puku. Aportes a la etnobotánica guaraní de algunas especies arbóreas del Paraguay Oriental. Centro de Estudios Antropológicos, Universidad Católica "Nuestra Señora de la Asunción”. 52 pp.

CADOGAN, L. 1992. Diccionario Mbya-GuaraníCastellano. Asunción: Biblioteca Paraguaya de Antropología. 211 pp.

CORREA, R. F. 2003. El complejo de las "canchalaguas" en Argentina. Tesis Ph. D., Universidad Nacional de la Plata, Buenos Aires.

D'ORBIGNY, A. 1945. Viaje a la América Meridional (1826-1833).Tomos I-II-III-IV. Editorial Futuro, Buenos Aires.

DI SAPIO, O., M. N CAMPAGNA, M. V. RODRÍGUEZ, M. L. MARTÍNEZ, S. GATTUSO, A. CORTADI \& M. GATTUSO. 2012. Parámetros micrográficos para la identificación de leño, corteza y hoja de Quassia amara L. (Simaroubaceae). Bol. Latinoam. Caribe Plant. Med. Aromat. 11: 172-187.

DOBRIZHOFFER, M. 1967 [1784]. Historia de los Abipones I. Resistencia: Facultad de Humanidades, Universidad Nacional del Nordeste. 568 pp.

FERNÁNDEZ DE OVIEDO, G. 1535. La historia general de las Indias. Sevilla, en la emprenta de Juan Crombergerm.

FONT QUER, P. 1993. Plantas medicinales. El Dioscorides renovado, tomo I. Barcelona: Editorial Labor S.A. 251 pp.

FURLONG, G. 1948. Naturalistas argentinos durante la dominación hispánica. Buenos Aires: Ed. Huarpes. $438 \mathrm{pp}$.

KELLER, H. A. 2007a. Notas sobre medicina y magia entre los guaraníes de Misiones, Argentina, un enfoque etnobotánico. Suplemento Antropológico de la Universidad Católica de Asunción 42: 345-384.

KELLER, H. A. 2007b. Origen mítico de la yerba mate, Ilex paraguariensis A.St.-Hil. (Aquifoliaceae), una versión mbya guaraní. Suplemento Antropológico de la Universidad Católica de Asunción 42: 335-344.
KELLER, H.A. 2008a. Thinouia mucronata (Sapindaceae), una especie ictiotóxica de los guaraníes de Misiones, Argentina. Bonplandia 17: 47-53.

KELLER, H. A. 2008b. Las plantas usadas en la construcción y acondicionamiento de viviendas y templos guaraníes en Misiones, Argentina. Bonplandia 17: 65-81.

KELLER H. A. 2008c. Etnobotánica de comunidades guaraníes de Misiones, Argentina; valoración de la vegetación como fuente de recursos. Tesis Ph. D., Universidad Nacional del Nordeste, Corrientes.

KELLER, H. A. 2011. Problemas de la etnotaxonomía guaraní: "las plantas de los animales". Bonplandia Ed. especial de Etnobotánica 20: 111-136.

KELLER, H. A. 2013. Árboles y arbustos en mitos sobre el origen y el fin del mundo de los guaraníes meridionales: elucidación de algunas expresiones fitonímicas. Bonplandia 22: 149-158.

KELLER, H. A. 2017. La Fotosíntesis de la Cultura, estudios etnobiológicos en comunidades guaraníes de Misiones, Argentina. Biblioteca Paraguaya de Antropología - Vol. 106. Asunción. 569 pp.

MONTENEGRO, P. DE. 2009. Materia medica misionera. Herbolario guaraní siglo XVII. Córdoba: Buena Vista Editores. 436 pp.

PAUCKE, F. 1944 [1749-1767]. Hacia allá y para acá (una estada entre los indios Mocobíes, 1749-1767), Tomo III, segunda parte. Universidad Nacional de Tucumán y Institución Cultural Argentino-Germana. TucumánBuenos Aires.

PIRONDO, A. 2016. Uso, Manejo y Conocimiento del Recurso Vegetal en Comunidades Rurales del Macrosistema Iberá" (Corrientes, Argentina). Tesis Ph. D., Universidad Nacional del Nordeste, Corrientes.

RATERA, E. \& M. RATERA. 1980. Plantas de la Flora argentina empleadas en Medicina popular. Buenos Aires: Hemisferio Sur. 189 pp.

SAGGESE, D. 1959. Plantas medicinales de Argentina. Rosario: Antognazzi \& Co. 189 pp.

TOURSARKISSIAN, M. 1980. Plantas medicinales de la Argentina. Buenos Aires: Hemisferio Sur. 178 pp.

ZULOAGA, F., O. MORRONE \& M. J. BELGRANO (EDS.). 2008. Catálogo de las plantas vasculares del Cono Sur (Argentina, Sur de Brasil, Chile, Paraguay y Uruguay). Disponible en: http://www2.darwin.edu.ar/ Proyectos/FloraArgentina/FA.asp. (Consulta: febrero de 2018).

Original recibido el 11 de abril de 2018; aceptado el 2 de mayo de 2018. 